\title{
EIN EINFACHES VERFAHREN Z.UR ALKYLIERUNG VON ARYLALKINEN
}

\author{
Manfred Lissel \\ Fakultät für Chemie, Universität Bielefeld, \\ Universitätsstr., D-4800 Bielefeld 1
}

Summary: Arylalkynes are alkylated in a rapid reaction and under mild conditions by alkyl lodides in the presence of powdered KOH and 18-crown-6

Die wichtigste Methode zur Herstellung von substituierten Alkinen ist die Alkylierung eines vorhandenen Alkins durch Alkylhalogenide. Zu den Standardmethoden für die Gewinnung des Acetylid-Anions gehört die Deprotonierung des Alkins durch Alkalimetall/flüssigen Ammoniak, Grignardverbindungen, Lithiumorganischen Verbindungen oder durch Alkalimetalle 1,2). Alkalimetallhydroxide werden als $z u$ schwache Basen eingestuft und dementsprechend nicht verwendet. Allein Phenylacetylen wurde mit Methyljodid in Gegenwart von KOH alkyliert: unter drastischen Reaktionsbedingungen bei $140^{\circ} \mathrm{C}$ im Bombenrohr erhielt man $30 \%$ 1-Phenyl-1-propin 3).

Die Alkylieruna von Phenylacetylen unter phasentransfer-katalytischen Bedingungen 4) wird seit langem diskutiert ${ }^{5)}$, gelang jedoch bisher nicht. Schwächere $\mathrm{CH}-\mathrm{Säuren}$ wie Fluoren ließen sich dagegen glatt alkylieren 6). Wir stellen hier eine phasentransfer-katalytische Variante vor, nach der die Alkylierung von aromatischen Alkinen in kurzer Zejt und unter schonenden Bedingungen durchgeführt werden kann. Dazu rührt man das Alkin in Benzol mit frisch gepulverter $\mathrm{KOH}$ und katalytischen Mengen 18-Krone-6 20 Minuten bei $80^{\circ} \mathrm{C}$ und versetzt mit Methyljodid. Nach weiteren 60 Minuten Rührzeit wird aufgearbeitet. Wir haben die in Tabelle 1 aufgefuhrten Acetylene erhalten, die angegebenen Ausbeuten sind die an isolierter Substanz. Die Anwendunosbreite dieser Methode wird zur Zeit untersucht, die Ergebnisse sollen in einer umfangrei- 
cheren Arbeit präsentiert werden.

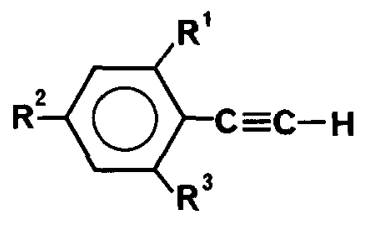

$1 a, b$

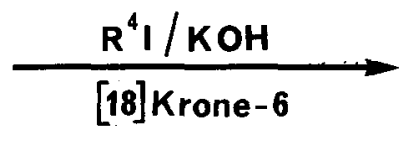

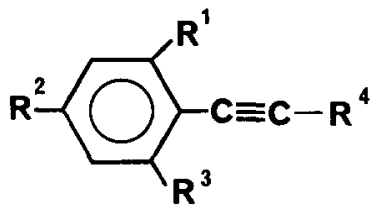

2 a-d

Tabe11e 1: Hergestellte Alkine

\begin{tabular}{l|lllll}
2 & $R^{1}$ & $R^{2}$ & $R^{3}$ & $R^{4}$ & Ausb.\% \\
\hline a & $\mathrm{H}$ & $\mathrm{H}$ & $\mathrm{H}$ & $\mathrm{CH}_{3}$ & 74 \\
b & $\mathrm{CH}_{3}$ & $\mathrm{CH}_{3}$ & $\mathrm{CH}_{3}$ & $\mathrm{CH}_{3}$ & 69 \\
c & $\mathrm{H}$ & $\mathrm{H}$ & $\mathrm{H}$ & $\mathrm{C}_{2} \mathrm{H}_{5}$ & 70 \\
d & $\mathrm{H}$ & $\mathrm{H}$ & $\mathrm{H}$ & $\mathrm{nC}_{8} \mathrm{H}_{17}$ & 63
\end{tabular}

Literatur und Anmerkungen:

1) W. Ziegenbein, in H.G. Viehe (Herausg.): Chemistry of Acetylens, S. 169, Marcel Dekker Inc., New York 1969.

2) V. Jäger in Houben-Weyl, Methoden der organischen Chemie, Bd. V/2a, S. 351, Thieme-Ver1ag, Stuttgart 1977.

3) J.U. Nef, Liebigs Ann. Chem. 310, 316 (1900).

4) E.V. Dehmlow, S.S. Dehmlow, Phase Transfer Catalysis, 2. Auf1., Verlag Chemie, Weinheim, 1983.

5) EUCHEM Conference: Phase Transfer Catalysis and related topics, Gargnano, Italien 1978.

6) M. Makosza, Bull. Acad. Pol. Sci. Ser. Sci. Chem. 15, 165 (1967).

(Received in Germany 24 January 1985) 